\title{
Photoplethysmography based instant remote monitoring of non-invasive blood pressure and oxygen saturation by using zigbee network.
}

\author{
Mehmet Merkepci $^{1}$, M. Sadettin Ozyazici ${ }^{2}$, Nuran Dogru ${ }^{1}$ \\ ${ }^{1}$ Department of Electrical and Electronics Engineering, University of Gaziantep, Gaziantep, Turkey \\ ${ }^{2}$ Department of Electrical and Electronics Engineering, Bahcesehir University, Istanbul, Turkey
}

\begin{abstract}
In this study, we measured blood pressure and blood oxygen saturation and monitored these vital signals remotely over a Zigbee network by using well-designed software having a Graphical User Interface. This paper proposes that measuring and transmitting these vital signals through a wireless network enables/ provides authorized staff to observe them remotely for instant intervention and doesn't discomfort the patient since the method's non-invasive photoplethysmography technique. Calculated blood pressure and oxygen saturation are displayed both on an LCD screen and a computer by means of a Zigbee module. The obtained results are compared to values from a pulse oximeter device to verify the accuracy of the designed device.
\end{abstract}

Keywords: Blood Pressure, Oxygen Saturation, Zigbee, Photoplethysmography, Non-invasive.

Accepted on August 11, 2017

\section{Introduction}

Photoplethysmography (PPG) is described as observing blood volume changes in the tissue [1]. PPG has widespread application in medical tools due to it is economy. Pulse oximeter can be given as an example of its application. In general, the PPG technique necessitates some opto-electronic components such as a source of light illuminate the tissue and a receiver (i.e photodetector) measuring variations in light intensity.

In general the PPG is used non-invasive form and peripheral pulse is well known waveform of the non-invasive PPG, and it is works synchronized to each heartbeat. In spite of the fact that the PPG signals are not completely understood, it is very simple and non-complex method. However, it is generally known that it can enlighten us about cardiovascular system [2].

This paper is structured in the following way. First, we describe the basic works principle of PPG, relation between the lights and tissue, and we review the root of PPG. Second, we investigate the instrumentation, measurement order, and pulse wave analysis. In addition, we study and design a new circuit which is capable of measuring $\mathrm{SpO}_{2}$ (oxygen saturation level) and heart rate, which is associated with clinical applications.

\section{Review and measurement of vital signals}

This section consists of two subsections. The first describes the measurement of blood oxygen saturation level (BOSL) and the second measurement of the blood pressure (BP).

\section{Measurement of blood oxygen saturation level}

For the past few decades, the pulse oximetry (PO) device has been very important device to observe clinical patient monitoring. The working principle of PO is based on the PPG technique. Using this technique we can obtain information about both the blood oxygen saturation level $\mathrm{SpO}_{2}$ and $\mathrm{BP}$ [3]. It is used for comprehensive practise in many different areas, such as hospital, clinics etc. Since 1990's the PO device has been a compulsory universal regulation for monitoring throughout anesthesia. We can refer the sudy by Kyriacou et al. [4] for good description of the technique as well as its basic operational principle, measurement technology and clinical applications.

Measurement of blood oxygen saturation level $\mathrm{SpO}_{2}$ or BOSL and blood pressure (BP) will be explained in detail in a later section. But, we briefly refer to the measurement of these parameters here. BOSL is measured by using red light and IR (infrared) light along vascular tissue, with quick exchange between the wavelengths. Changes in $\mathrm{SpO}_{2}$ affect the amplitudes of the red and infrared AC signals because the light absorption of $\mathrm{HbO}_{2}$ and $\mathrm{Hb}$ are different at these two wavelengths. BOSL is determined by using their amplitude ratio, and corresponding PPG DC components. This progression generally includes an empirically created calibration factor [5]. The pulsatile part from those PPG signal results from the blood vessel blood volume transforms for every pulse. If the peripheral pulse increases or decreases, the oxygen saturation level can be affected by dyshaemoglobinaemias, and low saturation levels can decrease its accuracy [6]. PO determines the blood oxygen level 
utilizing either the reflection or transmission modes of operation [7].

\section{Heart rate}

Heart rate (HR) is one of the most significant parameter among the vital signals that to measure for patient monitoring. The PPG pulse has both AC signal and DC signal. The AC signal component is related to the heart beat (HB) and so provides heart rate information. This information can be shown in PO. However, sometimes we can reduce confidence in the rate parameter because of a movement artefact or cardiac arrhythmia [2]. The reliability of reference heart rates can be found in quality index [8].

\section{Blood pressure}

BP which is generally known as arterial blood pressure [9]. BP is a very important indicator for human health and it is used to observe the physiological conditions of human in addition to other important signals like heart rate and blood oxygen saturation level. BP is classified in two categories. First is systolic blood pressure (SBP) and second is diastolic blood pressure (DBP). DBP is the minimum pressure on walls of the arterial whereas SBP is the maximum peak pressure. SBP and DBP may change during the day. Due to some factors such as stress, medicines, illness and exercises they can vary.

The human's BP is generally interpreted from the viewpoint of the SBP over DBP and it is determined in millimeters of mercury $(\mathrm{mm} \mathrm{Hg})$. In generally, normal BP is approximately $80 / 120 \mathrm{mmHg}$ for an adult. This value, however, changes depending on activity and medical condition by the nervous and other factors [10]. Low BP is known as hypotension and high is known as a hypertension [11]. They can range from moderate to severe, together with intense and chronic forms.

\section{Materials and Methods}

Oxygen saturation level and heart beat rate that can be measured invasively by piercing the wall of an artery to directly access the blood vessel [12]. This method is generally applied in a hospital or health instution. Invasive measurements are much harder and slower than non-invasive measurements. Therefore, non-invasive methods are more effective and easier than invasive ones and less painful for the patient. Although this method is convenient, it is not suitable for long-term use because it limits the range of motion of the person. A remote monitored non-invasive photoplethysmographic (PPG) technique has been developed to solve this problem.

\section{Measurement device}

Figure 1 demonstrates the block diagram of the designed device. Principally, the device consists of a high intensity red LED, infrared and a color sensor. The red LED and infrared is clipped on one side of the finger and the color sensor is clipped on the other side of finger. The color sensor emits infrared light and red light from the finger. This light is detected by the color sensor and the change of blood volume is measured through the finger artery. The color sensor transforms the PPG signal to a pulse signal which is then amplified and filtered accordingly. Then microcontroller evaluates all data for analysis and display. Finally, the microcontroller determines the number of pulses between defined time interval and so heart rate of the person is obtained.

The oxygen saturation level is determined by optoelectronic devices such as a color sensor or a photo detector. In this study we used a color sensor. Other important materials are red light and infrared (IR) light [13]. Oxygen which is carried by hemoglobin travels across the blood vessels. If hemoglobin carry the oxygen or not the absorbed light has different wavelengths and BOSL is calculated using these values as indicated in equation (1) [14].

The pulse oxymetric method is adopted in which the tissue is illuminated using the two LED's with two different wavelengths $(640 \mathrm{~nm}$ and $910 \mathrm{~nm})$ [15]. The percentage of saturated hemoglobin is determined by taking the ratio of the absorption of the two wavelengths.

Previous studies show that the oxy and deoxy hemoglobin have different optical attenuation characteristics. The best result is obtained by using the wavelengths at $640 \mathrm{~nm}(\mathrm{R})$ and $910 \mathrm{~nm}$ (IR). Since there is a color difference between oxygen bounded and unbounded hemoglobin, absorption percentage of red and infrared light is measured [16].

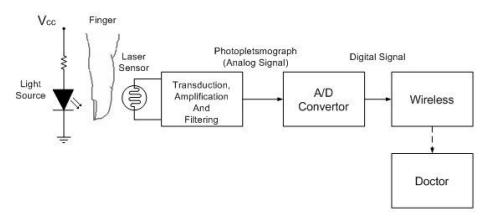

Figure 1. Block diagram of the designed device.

$\mathrm{SpO}_{2}=\frac{\mathrm{HbO}_{2}}{\mathrm{HbO}_{2}+\mathrm{Hb}} \times 100 \rightarrow(1)$

The pulse oxymeter uses Beer-Lambert's law. Transmitted light in solution is determined from the intensity of incident light [14]. $\mathrm{I}_{0}$ represents the intensity of transmitted light and IN represents intensity of incident light in equation (2).

$\mathrm{I}_{0}=\mathrm{INe}-\epsilon \mathrm{cL} \rightarrow(2)$

Here, $L$ represents the optical path length $(\mathrm{cm})$, c represents the concentration of the absorber and $\epsilon$ represents the wavelengthdependent extinction coefficient. In equation (3) Absorbance (A) can be expressed with regard to the absorbed light which passing through the solution.

$A=\ln \left(\frac{I_{0}}{I_{n}}\right)=\in c L \rightarrow(3)$

Absorbance (A) which is termed as optical density (OD) commonly. If the wavelength extinction coefficient and optical length are known, Beer's Lambert's law can be used to determine the concentration. To determine the value of SPO2, 
firstly we should determine the value of $\mathrm{R}$ which depends on the DC and AC components of the PPG signal. Namely, to determine the R value DCR, DCIR, ACR and ACIR must be determine respectively. Then normalized red to IR absorption ratio is given as in equation (4)

$$
R=\frac{\frac{A C_{R}}{D C_{R}}}{\frac{A C_{I R}}{D C_{I R}}} \rightarrow(4)
$$

If we determine the value of $\mathrm{R}$ then we can calculate the value of $\mathrm{SpO}_{2}$ using equation (5) [16].

$\% \mathrm{SpO}_{2}=\mathrm{K} * \mathrm{R} \rightarrow(5)$

Here, $\mathrm{K}$ is used to increase the accuracy of measured results.

Also, a remote monitored system is added to the designed system to allow an authorized person to observe measurements.

\section{Working principle}

The device has a menu to measure $\mathrm{SpO}_{2}$ level and Heart beat (HB) and by using this menu the vital parameters can be measured together or separately. The menu is shown in Figure 2.

Basically, the designed device uses light to measure oxygen saturation level. Two LEDs emit light at two different wavelengths at the device's probe which illuminates the tissue and the detector behind the tissue detects the absorbed light. When a finger is placed between LEDs and detector, some amount of light reaches to detector which causes device to measure the oxygen saturation level and blood pressure. Based on these measurements, the physical condition of persons can be determined.

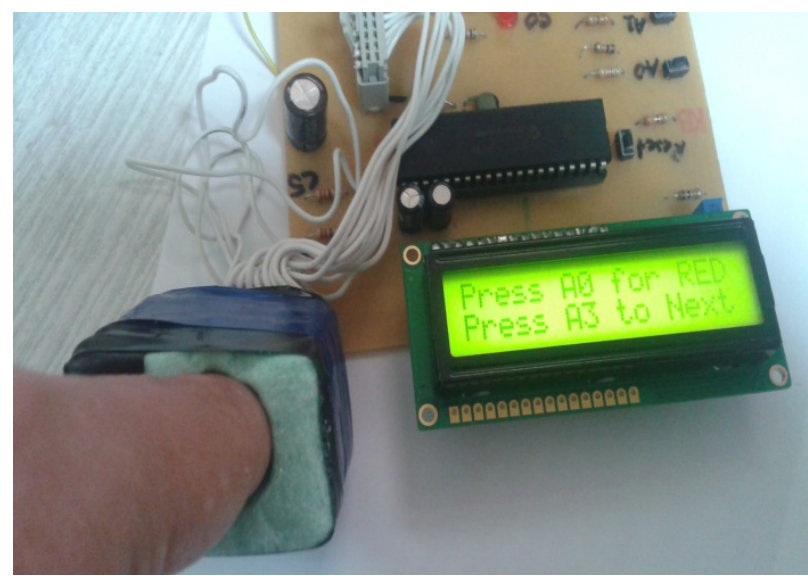

Figure 2. Designed probe and device.

\section{Results and Discussions}

The designed PPG sensor comprises an IR (910 nm), RED LED $(640 \mathrm{~nm})$ and color sensor (TSL230R) combination. Color sensor is used collect to reflectance light from finger. It converts light to frequency (square wave) that is directly proportional to the incident light intensity. Using this feature, the $\mathrm{SpO}_{2}$ and pulse rate are calculated accurately with real time. Microcontroller is used PIC16F877A. The microcontroller is programmed to take reading of the color sensor's RED and IR values every second. Microcontroller evaluates Heart Rate and $\mathrm{SPO}_{2}$ values. The next step is to make the proposed device wireless. To do this 2 zigbee modules are used as a receiver and transmitter. Measured values are sent by using zigbee module and measurement is completed. Our experiment results confirm that our proposed $\mathrm{SpO}_{2}$ monitoring is accurate. Our device is tested on 10 healty persons and measurements results are compared with a standard PulseOximeter device. It can be shown in Table1. Heart Rate measurements ranged in percent error between $2-4 \%$. For $\mathrm{SpO}_{2}$ a low percent error was maintained for all the constant measurements. To increase a more precise accuracy of device, further testing should be done.

Table 1. Experimental values for different persons.

\begin{tabular}{lllll}
\hline Persons & $\begin{array}{l}\mathrm{SpO}_{2} \\
\text { Our Results }\end{array}$ & $\begin{array}{l}\text { Heart Rate } \\
\text { Our Results }\end{array}$ & $\begin{array}{l}\mathrm{SpO}_{2} \\
\text { Device Results }\end{array}$ & $\begin{array}{l}\text { Heart Rate } \\
\text { Device Results }\end{array}$ \\
\hline 1 & 98,32 & 89 & 99 & 91 \\
\hline 2 & 96,04 & 94 & 97 & 95 \\
\hline 3 & 97,52 & 96 & 98 & 96 \\
\hline 4 & 94,27 & 98 & 95 & 99 \\
\hline 5 & 91,63 & 90 & 92 & 92 \\
\hline 6 & 95,18 & 94 & 93 & 93 \\
\hline 7 & 98,13 & 97 & 98 & 97 \\
\hline 8 & 90,36 & 88 & 91 & 89 \\
\hline 9 & 92,48 & 96 & 93 & 95 \\
\hline 10 & 94,55 & 100 & 95 & 101 \\
\hline
\end{tabular}

\section{Conclusions}

In this paper, a low-cost microcontroller based pulse oxymeter is designed. The device has advantages such as it can be used by nonprofessional people at home to measure the heart rate and blood oxygen saturation easily and can be monitored by a person for example doctor or authorized person. Measurements can be done at home or outside of the home. Results can be logged onto the device and then send to a computer of related persons using zigbee modules. This is great significance for the elder people to measure of oxygen saturation and heart rate.

\section{References}

1. Challoner AVJ. Photoelectric plethysmography for estimating cutaneous blood flow, in $\mathrm{P}$ Rolfe, Ed, noninvasive physiological measurements (1 vol). Academic Press, London, UK 1979; 127.

2. Allen J. Photoplethysmography and its application in clinical physiological measurement. Physiol Meas 2007; 28: 1-39. 
3. Aoyagi T, Miyasaka K. Pulse oximetry: its invention, contribution to medicine, and future tasks. Anesth Analg 2002; 94: 1-3.

4. Kyriacou PA, Pal SK, Langford R, Jones DP. Electrooptical techniques for the investigation of oesophageal photoplethysmographic signals and blood oxygen saturation in burns. Measurement Control 2006; 39: 77-79.

5. Webster JG. Design of pulse oximeters. Institute of Physics Pub, Philadelphia 1997.

6. Kyriacou AP. Functional, overlapping, competing, jurisdictions and ethnic conflict management. Kyklos 2006; 159: 63-83.

7. Mendelson Y, Ochs BD. Non-invasive pulse oximetry utilizing skin reflectance photoplethysmography. IEEE Transac Biomed Eng 1988; 35: 798-805.

8. Yu H, Kaufman YJ, Chin M, Feingold G, Remer LA, Anderson TL, Balkanski Y, Bellouin N, Boucher O, Christopher S, DeCola P, Kahn R, Koch D, Loeb N, Reddy MS, Schulz M, Takemura T, Zhou M. A review of measurement-based assessment of aerosol direct radiative effect and forcing. Atmos Chem Phys 2006; 6: 613-666.

9. Webster JG. Medical instrumentation, application and design (2 ed). Houghton Mifflin Company, USA 1992.

10. Darovic, Gloriya O. Hemodynamic monitoring: Invasive and non-invasive clinical application (2 ed). WB Saunders Company, Philadelphia, 1995.

11. US Department Of Health And Human Services. Diagnosis, evaluation, and treatment of high blood pressure in children and adolescents. National Institutes of Health National 2003; 03-5232.

12. Lindberg LG, Oberg PA. Photoplethysmography: Influence of light source wavelength. Med Biol Eng Comput 1991; 29: 48-54.

13. Lindberg LG, Oberg PA. Optical properties of blood in motion. Opt Eng 1993; 33: 253-257.

14. Gazi Maruf A, Adel Al-J, Mohamed Al J. Continuous measurement of oxygen saturation level using photoplethysmograph signal. Int Conf Biomed Pharm Eng ICBPE 2006.

15. Alrick BH. The blood supply of various skin areas as estimated by the photoelectric plethysmograph. Am J Physiol 1938; 124: 328-340.

16. Anan W, Amphawan J, Prasit J. Continuous Measurements of ecg and spo2 for cardiology information system. Proc Int Multi-Conf Eng Comp Sci 2009; 2: 18-20.

\section{*Correspondence to}

Mehmet Merkepci

Department of Electrical and Electronics

University of Gaziantep

Gaziantep

Turkey 